\title{
Group Decision Making: Consensus Approaches based on Soft Consensus Measures
}

\author{
Francisco Javier Cabrerizo, Ignacio Javier Pérez, Francisco Chiclana and \\ Enrique Herrera-Viedma
}

\begin{abstract}
A group decision making situation involves multiple decision makers communicating with others to reach a decision. In such a situation, the most important issue is to obtain a decision that is best acceptable by the decision makers, and, therefore, consensus has attained a great attention and it is a major goal of group decision making situations. To measure the closeness among the opinions given by the decision makers, different approaches have been proposed. At the beginning, consensus was meant to be a unanimous and full agreement. However, because this situation is often not reachable in practice, the use of a softer consensus, which assesses the level of agreement in a more flexible way and reflects the large spectrum of possible partial agreements, is a more reasonable approach. Soft consensus approaches better reflects a real human perception of the essence of consensus and, therefore, they have been widely used. The purpose of this contribution is to review the different consensus approaches based on soft consensus measures that have been proposed.
\end{abstract}

Keywords Group decision making · Consensus · Fuzzy set theory

F.J. Cabrerizo (四) · E. Herrera-Viedma

Department of Computer Sciences and Artificial Intelligence,

University of Granada,

C/ Periodista Daniel Saucedo Aranda s/n, 18071 - Granada Spain

e-mail: cabrerizo@decsai.ugr.es,viedma@decsai.ugr.es

I.J. Pérez

Department of Computer Sciences and Engineering,

University of Cádiz,

Avenida Universidad de Cádiz 10, 11519 - Puerto Real, Spain

e-mail: ignaciojavier.perez@uca.es

F. Chiclana

Centre for Computational Intelligence, Faculty of Technology,

De Montfort University,

Leicester, LE1 9BH, UK

e-mail: chiclana@dmu.ac.uk 


\section{Introduction}

One of the most crucial human activities is decision making, which consists of finding the best alternative, variant, opinion, and so on, from among some possible ones. This task generally involves multiple decision makers to make the decision $[10,34,47]$ and, then, it is called a multiperson or group decision making (GDM) situation [34].

In a GDM situation there is a group of decision makers expressing their preferences or opinions concerning a set of different alternatives. In such a context, the question is to find a solution which is best acceptable by the whole group of decision makers. Here, the process arriving at an agreed-upon opinion, perhaps consensus, by using in a democratic way knowledge of the different decision makers, leads to better decision [5].

The term consensus has been used for centuries in different areas and contexts. When it is used in GDM contexts, an important issue is the very meaning of consensus and the problems related to its essence.

First, consensus makes reference to the state of accordance with a group of decision makers in the sense that they show similar preferences or opinions related to the alternatives in question. In this sense, consensus was initially meant as a complete agreement. In such a way, some authors proposed consensus measures assuming values in-between 0 , meaning no consensus or partial consensus, and 1 , meaning full consensus [3, 51]. The above situation has however been considered impractical in most real world situations as decision makers on rare occasions arrive at that complete agreement. Therefore, the very essence of consensus was reconsidered, and it was admitted that the decision makers are not willing to fully change their preferences or opinions so that the consensus will not be a complete agreement. A milestone was here a special issue of the Synthese journal [40]. In particular, the paper written by Loewer and Laddaga [41] is the most relevant for this purpose, in which, these authors clearly made the case for a soft concept of consensus stating that:

"... It can correctly be said that there is a consensus among biologists that Darwinian natural selection is an important cause of evolution though there is currently no consensus concerning Gould's hypothesis of speciation. This means that there is a widespread agreement among biologists concerning the first matter but disagreement concerning the second ..."

It was suggested that a fuzzy majority is suitable, and that it makes sense to speak about a degree of consensus, or a distance from ideal consensus. The linguistic quantifiers, exemplified, for example, by 'most", "almost all", "much more than a half", and so on, are a natural manifestation of this fuzzy majority. Linguistic quantifiers can be handled by a calculus of linguistically quantified propositions [56], and also by using aggregation operators or aggregation funcions [20, 53], in particular, Yager's OWA (Ordered Weighted Average) operators [54], which offer a much needed generality and flexibility [57]. Janusz Kacprzyk introduced the concept of a fuzzy majority related to a fuzzy linguistic quantifier into GDM situations 
$[32,33,34]$. Since then, the concept of a fuzzy majority has been the key point for new definitions of soft consensus [36, 37, 6, 31], which assess the degree of agreement in a more flexible way, reflecting the large spectrum of possible partial agreements and guiding the discussion process until widespread agreement, not always full, is achieved among the decision makers.

Second, consensus refers as a process to reach agreement. This process involves an evolution of the preferences expressed by the decision makers towards agreement with respect to their preferences. In such a situation, the point of departure is a the set of preferences given by the particular decision makers concerning in general opinions as to the values of some quantities. At the beginning, the preferences expressed by the decision makers was equated with some utilities resulting from some courses of actions, the probabilities of them, and alike [12, 18, 22]. Nevertheless, since GDM situations are centered on decision makers, coming with inherent subjectivity, imprecision and vagueness in the articulation of preferences, the theory of fuzzy sets [55], has delivered new tools in this field for a long time, as it is a more adequate tool to represent often not clear-cut human preferences encountered in most practical cases. Fuzzy logic has played here a considerable role by providing means for the representation and processing of imprecise information and preferences [17].

Because it is important to obtain an approved solution by all the decision makers, the consensus is one of the major goals of GDM situations. Concretely, consensus approaches based on soft consensus measures have been widely proposed in the literature $[6,31]$, as it is more human-consistent and suitable for reflecting human perceptions of the meaning of consensus.

The objective of this contribution is to review the different consensus approaches based on soft consensus measures that have been proposed in the literature. To do so, the pioneering contributions are described and a comprehensive presentation of the state of the art of all kinds of consensus related problems is shown. After some decades of fruitful research in this field, it is a good time for looking backward an review what the research has been developed on this topic.

This contribution is outlined as follows. In Section 2, we introduce the GDM framework and the usual consensus process. Section 3 highlights the pioneering and most important contributions existing on consensus approaches based on soft consensus measures. In Section 4, we describe the main consensus approaches based on soft consensus measures. Finally, in Section 5, we present some conclusions and future work.

\section{Preliminaries}

This section is devoted to introduce the GDM framework to develop consensus processes. Concretely, the GDM situation is defined, the formats of preferences utilized by the decision makers to provide their opinions are presented, and the usual consensus process is described. 


\subsection{GDM Framework}

There have been several efforts in the specialized literature to create different models to correctly address and solve GDM situations. Some of them make use of fuzzy set theory as it is a good tool to model and deal with vague or imprecise opinions $[19,23,38,48]$.

In a classical GDM situation [9, 19, 34], there is a problem to solve, a solution set of possible alternatives, $X=\left\{x_{1}, x_{2}, \ldots, x_{n}\right\}(n \geq 2)$, and a group of two or more decision makers, $E=\left\{e_{1}, e_{2}, \ldots, e_{m}\right\}(m \geq 2)$, characterized by their background and knowledge, who express their opinions about the alternatives to achieve a common solution. In a fuzzy context, the objective is to classify the alternatives from best to worst, associating with them some degrees of preference expressed in the $[0,1]$ interval.

Decision makers can use several preference representation structures to provide their preferences or opinions about the alternatives in a GDM situation. The most common ones that have been widely used in the literature are the following:

- Preference orderings. Using this preference representation structure, the opinions of a decision maker $e_{l} \in E$ about a set of feasible alternatives $X$ are described as a preference ordering $O^{l}=\left\{o_{1}^{l}, \ldots, o_{n}^{l}\right\}$, where $o^{l}(\cdot)$ is a permutation function over the indexes set $\{1, \ldots, n\}$ [52]. Hence, a decision maker gives an ordered vector of alternatives from best to worst.

- Utility values. Using this preference representation structure, a decision maker $e_{l} \in E$ expresses his/her opinions about a set of feasible alternatives $X$ by means of a set of $n$ utility values $U^{l}=\left\{u_{1}^{l}, \ldots, u_{n}^{l}\right\}, u_{i}^{l} \in[0,1]$. Here, the higher the value for an alternative, the better it satisfies decision maker's objective [28].

- Preference relations. In this case, the preferences given by the decision maker on $X$ are described by a function $\mu_{P^{l}}: X \times X \rightarrow D$ where $\mu_{P l}\left(x_{i}, x_{j}\right)=p_{i j}^{l}$ can be interpreted as the preference degree or intensity of the alternative $x_{i}$ over $x_{j}$ expressed in the information representation domain $D$. Different types of preference relations can be used according to the domain used to evaluate the intensity of the preference:

1. Fuzzy preference relations [34]: If $D=[0,1]$, every value $p_{i j}^{l}$ in the matrix $P^{l}$ represents the preference degree or intensity of preference of the alternative $x_{i}$ over $x_{j}: p_{i j}^{l}=1 / 2$ indicates indifference between $x_{i}$ and $x_{j}, p_{i j}^{l}=1$ indicates that $x_{i}$ is absolutely preferred to $x_{j}$, and $p_{i j}>1 / 2$ indicates that $x_{i}$ is preferred to $x_{k}$. It is usual to assume the additive reciprocity property $p_{i j}^{l}+p_{j i}^{l}=1 \forall i, j$.

2. Multiplicative preference relations [49]: If $D=[1 / 9,9]$, and then every value $p_{i j}^{l}$ in the matrix $P^{l}$ represents a ratio of the preference intensity of the alternative $x_{i}$ to that of $x_{j}$, i.e., it is interpreted as $x_{i}$ is $p_{i j}^{l}$ times good as $x_{j}: p_{i j}^{l}=1$ indicates indifference between $x_{i}$ and $x_{j}, p_{i j}^{l}=9$ indicates that $x_{i}$ is unanimously preferred and $p_{i j}^{l} \in\{2,3, \ldots, 8\}$ indicates intermedi- 
ate evaluations. It is usual to assume the multiplicative reciprocity property $p_{i j}^{l} \cdot p_{j i}^{l}=1 \forall i, j$ too.

3. Linguistic preference relations $[23,24]$ : If $D=S$, where $S$ is a linguistic term set $S=\left\{s_{0}, \ldots, s_{g}\right\}$ with odd cardinality $(\mathrm{g}+1), s_{g / 2}$ being a neutral label (meaning "equally preferred") and the rest of labels distributed homogeneously around it, then every value $p_{i j}^{l}$ in the matrix $P^{l}$ represents the linguistic preference degree or linguistic intensity of preference of the alternative $x_{i}$ over $x_{j}$.

Among the different representation formats that decision makers may use to provide their opinions, fuzzy preference relations $[34,44]$ are one of the most used because of their effectiveness as a tool for modelling decision processes. In particular, they are very useful when we want to aggregate decision makers' preferences into a collective one $[34,52]$, which is carried out by using aggregation functions or aggregation operators $[20,53]$.

Finally, according to the importance of each decision maker, GDM situations are usually classified into two groups $[11,46]$ :

- A GDM situation is heterogeneous when the opinions of the decision makers are not equally important.

- A GDM situation is homogeneous if every opinion is treated equally.

A way to implement this heterogeneity is to assign a weight to every decision maker. Weights are qualitative or quantitative values that can be assigned in several different ways [11]: (i) weights can be assigned directly, (ii) or they can be obtained automatically from the opinions provided by the decision makers. The weights can be interpreted as a fuzzy subset, $I$, with a membership function, $\mu_{I}: E \rightarrow[0,1]$, in such a way that $\mu_{I}\left(e_{l}\right) \in[0,1]$ denotes the importance degree of the decision maker within the group, or how relevant is the decision maker in relation with the problem to be solved $[14,15]$. Finally, it should be pointed out that fuzzy measures and fuzzy integrals $[21,43]$ can also be used to implement the heterogeneity among the decision makers.

\subsection{Consensus Process}

A way of solving GDM situations is by carrying out a selection process to choose a solution set of alternatives from the opinions provided by the decision makers $[19,48]$, without taking into account the level of agreement. It involves two different steps [7, 48]: (i) aggregation of individual preferences, and (ii) exploitation of the collective preference. However, this process can lead sometimes solutions that are not well accepted by some decision makers in the group [5, 50], because they could consider that their iopinions have not been considered properly to obtain the solution, and, hence, they might reject it. To avoid this situation, it is advisable that decision makers carry out a consensus process. For this reason, GDM problems are 
usually faced by applying a consensus process and a selection process before a final solution can be obtained [38].

Two approaches may be distinguished in the formulation of a consensus process. The traditional one, in which the process is modeled by using matrix calculus or Markov chains to model the time evolution of changes of opinions toward consensus $[12,18,22]$. The approaches exemplified by the above citations have contributed much to the understanding of the process and its dynamics. However, it has been considered much more promising to run the consensus process with the help of a special agent, called a moderator, whose task is to help the decision makers involved while changing their testimonies towards consensus, by rational argument, persuasion, and so on. This second approach, in which there is a moderator, is more promising in practice and the most used.

According to this second approach, a consensus process is an iterative process composed of several consensus rounds, where the decision makers accept to change their opinions following the advice given by a moderator, which knows the agreement in each moment of the consensus process by means of the computation of some consensus measures.

\section{Pioneering Contributions}

In this section, the innovative and prominent contributions in the field of consensus approaches based on soft consensus measures are revised. As we have aforementioned, people are generally willing to accept that consensus has been reached when most actors agree with the opinions associated with the most relevant alternatives. The milestone was a special issue published in the Synthese journal:

B. Loewer. Special issue on consensus. Synthese 62 (1), 1-122 (1985).

Among many papers therein, Loewer and Laddaga wrote the most important one for our purpose:

B. Loewer, R. Laddaga. Destroying the consensus. Synthese 62 (1), 79-96 (1985).

Here the first approach for a soft concept of consensus was clearly made, suggesting that a fuzzy majority is appropriate, and that it makes sense to speak about a degree of consensus, or a distance from (ideal) consensus.

According to Loewer and Laddaga, Kacprzyk and Fedrizzi introduced the concept of a fuzzy majority using Zadeh's fuzzy linguistic quantifier to compute soft consensus measures in the following prominent contributions:

J. Kacprzyk, M. Fedrizzi. Soft consensus measure for monitoring real consensus reaching processes under fuzzy preferences. Control and Cybernetics 15 (3-4), 309-323 (1986).

J. Kacprzyk, M. Fedrizzi. A 'soft' measure of consensus in the setting of partial (fuzzy) preferences. European Journal of Operational Research 34 (3), 316-325 (1988).

J. Kacprzyk, M. Fedrizzi. A 'human-consistent' degree of consensus based on fuzzy logic with linguistic quantifiers. Mathematical Social Sciences 18 (3), 275-290 (1989). 
Then, the classical operational definition of consensus was expressed by a linguistically quantified proposition as:

"Most $(Q 1)$ of the important $(B)$ individuals agree as to almost all ( $Q 2)$ relevant $(I)$ alternatives”

where: $Q 1$ and $Q 2$ are fuzzy linguistic quantifiers [56], e.g.,"most" and "almost all", and $B$ and $I$ stand for fuzzy sets denoting the importance/relevance of the individuals and alternatives.

The above works constituted the basis of many consensus approaches based on soft consensus measures proposed later. In the following, some of the most prominent contributions are presented:

- Herrera, Herrera-Viedma and Verdegay defined the first soft consensus model in GDM problems in a fuzzy linguistic context:

F. Herrera, E. Herrera-Viedma, J. L. Verdegay, A model of consensus in group decision making under linguistic assessments. Fuzzy Sets and Systems 78 (1), 73-87 (1996).

This prominent contribution has shown a high impact in the fuzzy decision making community, and it is considered a highly cited paper according to the Essential Science Indicators (ESI) database, published by Thomson Reuters. Here, the authors present a new consensus model for GDM problems based on fuzzy linguistic preference relations defined in an ordinal fuzzy linguistic approach $[26,27]$. As main novelty, two types of soft consensus measures to guide the consensus process are defined: (i) consensus degrees, and (ii) proximity measures. In addition, they are applied in three activity levels: (i) level of preference, (ii) level of alternative, and (iii) level of preference relation. The consensus degrees indicate how far a group of decision makers is from the maximum consensus, and the proximity measures indicate how far each decision maker is from current consensus labels over the preferences. In such a way, the moderator is provided with a complete consensus instrument to control the consensus process.

- Later, assuming also a fuzzy linguistic context, the same authors presented the first consensus model which is guided by both consensus and consistency measures:

F. Herrera, E. Herrera-Viedma, J. L. Verdegay. A rational consensus model in group decision making using linguistic assessments. Fuzzy Sets and Systems 88 (1), 31-49 (1997).

In this new approach, the moderator is provided with consistency measures to guide the consensus process too. This consensus approach offers the possibility of achieving more rational consensus solutions, i.e., less distorted consensus solutions due to inconsistencies in the decision makers' preferences.

- Other prominent contribution in soft consensus was proposed by HerreraViedma, Herrera, and Chiclana: 
E. Herrera-Viedma, F. Herrera, F. Chiclana. A consensus model for multiperson decision making with different preference structures. IEEE Transactions on Systems Man and Cybernetics-Part A: Systems and Humans 32 (3), 394-402 (2002).

In this consensus approach, the decision makers can provide their preferences with different preference representation structures. Two main novelties are also contained in this contribution. Firstly, soft consensus measures are computed by comparison between decision makers' solutions and not between decision makers' preferences, as it usually happens in previous consensus approaches. In such a way, the problem of computing consensus measures is overcome when we use different preference representation structures in GDM problems. And secondly, using these measures, a feedback mechanism based on simple and easy rules to help decision makers change their preferences is defined. Therefore, the consensus process could be guided automatically, without a moderator, avoiding the possible subjectivity that he/she could introduce into the process. We should point out that this consensus contribution is a highly cited paper according to the ESI database too.

- Herrera-Viedma, Martinez, Mata and Chiclana dealt with the consensus problem when the GDM problem is defined in a fuzzy multi-granular linguistic context, i.e., by assuming that decision makers could use different linguistic term sets to provide their preferences:

E. Herrera-Viedma, L. Martinez, F. Mata, F. Chiclana. A consensus support system model for group decision-making problems with multigranular linguistic preference relations. IEEE Transactions on Fuzzy Systems 13 (5), 644-658 (2005).

The main novelty of this contribution is to present an automatic control system to guide the consensus process that substitutes the moderator's actions. To do so, this approach uses the consensus degrees to decide when the consensus process should finish and the proximity measures to define a recommendation system that recommends decision makers about the preferences that they should change in the next consensus rounds. This contribution is also considered a highly cited paper according to the ESI database.

- Finally, other seminal consensus contribution was proposed by Herrera-Viedma, Alonso, Chiclana, and Herrera in:

E. Herrera-Viedma, S. Alonso, F. Chiclana, and F. Herrera. A consensus model for group decision making with incomplete fuzzy preference relations. IEEE Transactions on Fuzzy Systems 15 (5), 863-877 (2007).

The main novelty of this soft consensus approach is that it provides tools to support the consensus processes in the presence of missing values or incomplete information in GDM situations. Here, the authors define the first consensus approach based on soft consensus measures which is carried out automatically (without a moderator) by three kinds of measures: consensus measures, consistency measures and incompleteness measures, too. Similarly, this contribution is considered a highly cited paper in the ESI database. 
Finally, the main novelties of the above prominent consensus approaches based on soft consensus measures are summarized in Table 1.

Table 1 Most prominent soft consensus approaches

Contribution Novelties

F. Herrera, E. Herrera-Viedma, J. L. Verdegay, A model of consensus in group decision making under linguistic assessments. Fuzzy Sets and Systems 78 (1), 73-87 (1996)

- It defines the first soft consensus approach in a fuzzy linguistic context

- It uses both consensus degrees and proximity measures to guide the consensus process

F. Herrera, E. Herrera-Viedma, J. L. Verdegay. A rational consensus model in group decision making using linguistic assessments. Fuzzy Sets and Systems 88 (1), 31-49 (1997).

- It is guided by both consensus and consistency measures

E. Herrera-Viedma, F. Herrera, F. Chiclana. A consensus model for multiperson decision making with different preference structures. IEEE Transactions on Systems Man and CyberneticsPart A: Systems and Humans 32 (3), 394-402 (2002)

- Different preference representation structures can be used

- Soft consensus measures are computed by comparison between decision makers' solutions

- A feedback mechanism is incorporated

E. Herrera-Viedma, L. Martinez, F. Mata, F. Chiclana. A consensus support system model for group decision-making problems with multigranular linguistic preference relations. IEEE Transactions on Fuzzy Systems 13 (5), 644-658 (2005)

E. Herrera-Viedma, S. Alonso, F. Chiclana, and F. Herrera. A consensus model for group decision making with incomplete fuzzy preference relations. IEEE Transactions on Fuzzy Systems 15 (5), 863-877 (2007)

- It is defined in a fuzzy multi-granular linguistic context

- It presents an automatic control system substituting the moderator's actions

- It supports consensus processes in the presence of incomplete information

\section{Consensus Approaches Based on Soft Consensus Measures}

Consensus approaches based on soft consensus measures have been a hot topic in recent years [31], and different approaches can be found in the literature according to different criteria: (i) reference domain used to compute soft consensus measures, (ii) coincidence concept used to compute the soft consensus measures, (iii) generation method of recommendations, and (iv) guiding measures.

In the following subsections, these different consensus approaches are described in more detail. 


\subsection{Consensus Approaches Based on the Reference Domain}

Two different consensus approaches can be found according to the reference domain utilized to compute the consensus measures.

Firstly, consensus measures focused on the decision maker set have been presented in $[34,36,37,16]$, in which consensus measures are computed in three steps: (i) for each pair of decision makers, a degree of agreement as to their preferences between all the pair of alternatives are computed, (ii) these degrees are aggregated to obtain a degree of agreement of each pair of decision makers as to their preferences between $Q 1$ pairs of alternatives, and (iii) these degrees are aggregated to obtain a degree of agreement of $Q 2$ pairs of decision makers as to their preferences between $Q 1$ pair of alternatives, which is the degree of consensus sought.

Secondly, consensus measures focused on the alternative set have been presented in $[23,24,29,30]$, in which the consensus measures are computed at the three different levels of representation of a preference relation: (i) level of preference, indicating the consensus degree existing among all the $m$ preference values attributed by the $m$ decision makers to a specific preference, (ii) level of alternative, which allows us to measure the consensus existing over all the alternative pairs where a given alternative is present, and (iii) level of preference relation, which evaluates the social consensus, that is, the current consensus existing among all the decision makers about all the preferences. It allows us, for example, to identify which decision makers are close to the consensus solutions, or in which alternatives the decision makers are having more trouble to reach consensus.

Comparing both approaches, the latter seems better to design consensus processes allowing us to guide the decision makers to modify their opinions during the discussion process.

\subsection{Consensus Approaches Based on the Coincidence Method}

In the literature, we can find soft consensus measures valued in $[0,1]$, where a value close to 1 indicates a high level of consensus and a value close to 0 indicates a low level of consensus $[38,34,36,30,4]$. On the other hand, instead of using numerical values in $[0,1]$, soft consensus measures based on linguistic labels have been proposed $[24,25]$ to evaluate the level of consensus. Anyway, to obtain the level of consensus achieved in each round of the consensus process, the similarity among the preferences provided by the decision makers on the alternatives is measured. Soft consensus measures are based on the coincidence concept [25], and we can identify three different methods for computing them [7]:

1. Consensus measures based on strict coincidence among preferences [24, 35]. Here, similarity criteria among preferences are used to compute the coincidence concept. In such a case, only two possible results are assumed: 1 if the opinions are equal and, otherwise, a value of 0 . The advantage of this approach is that the 
computation of the consensus degrees is simple and easy. The drawback of this approach is that the consensus degrees obtained do not reflect the real consensus situation.

2. Consensus measures based on soft coincidence among preferences [1, 29, 34, 36]. As above, similarity criteria among preferences are used to compute the coincidence concept, but, now, a major number of possible coincidence degrees is considered. It is assumed that the coincidence concept is a gradual concept assessed with different degrees defined in the unit interval $[0,1]$. The advantage of this approach is that the consensus degrees obtained reflect better the real consensus situation. The drawback of this approach is that the computation of the consensus degrees is more difficult because we need to define similarity criteria to compute the consensus measures, and, sometimes it is not possible to define them directly.

3. Consensus measures based on coincidence among solutions [28, 2]. Here, similarity criteria among the solutions obtained from the decision makers' preferences are used to compute the coincidence concept and different degrees assessed in $[0,1]$ are assumed. The advantage of this approach is that the consensus degrees are obtained comparing not the opinions but the position of the alternatives in each solution, what allows us to reflect the real consensus situation in each moment of the consensus reaching process. The drawback of this approach is that the computation of the consensus degrees is more difficult than in the above approaches because we need to define similarity criteria and it is necessary to apply a selection process before obtaining the consensus degrees.

It should be pointed out that the second and third methods, which reflect the real consensus state within the group of decision makers [6], are the most useful approaches to design consensus processes allowing us to advice the decision makers during the consensus process [31]. In particular, the second method is applied in contexts under preference relations and the third one is applied in decision situations under different formats of preference representation.

\subsection{Consensus Approaches Based on the Generation Method of Recommendations}

The generation method of recommendations to the decision makers is very important in order to increase the consensus level. From this point of view, the first consensus approaches proposed in the literature [23, 24, 34, 36, 38] can be considered as basic approaches based on a moderator, who monitors the agreement in each moment of the consensus process and is in charge of supervising and addressing the consensus process towards success. However, the moderator can introduce some subjectivity in the process. 
To overcome this drawback, consensus approaches have been proposed by substituting the moderator figure or providing moderator with better analysis tools, making more effective and efficient the decision making processes:

- Consensus approaches incorporating a feedback mechanism substituting the moderator's actions have been developed [28, 30, 29]. In these approaches, proximity measures are calculated to evaluate the proximity between the individual decision makers' preferences and the collective one. These proximity measures allow to identify the preference values provided by the decision makers that are contributing less to reach a high consensus state. In such a way, the feedback mechanism gives advice to those decision makers to find out the changes they need to make in their opinions to obtain a solution with a better consensus degree.

- Consensus approaches have been proposed using a novel data mining tool [39], the so called action rules [45], to stimulate and support the discussion in the group. The purpose of an action rule is to show how a subset of flexible attributes should be changed to obtain an expected change in the decision attribute for a subset of objects characterized by some values of the subset of stable attributes. According to it, these action rules are used to indicate and suggest to the moderator with which decision makers and with respect to which options it may be expedient to deal.

It should be pointed out that the current consensus trends are committed to develop automated feedback mechanisms replacing the moderator, in particular, when consensus processes are developed in crowded social environments [1]. In addition, new feedback mechanisms which implement strategies that adjust the number of changes required depending on the level of consensus among decision makers in each consensus round are being proposed [42].

\subsection{Consensus Approaches Based on Guidance Measures}

The pairwise comparison in preference relations helps the decision makers to provide their preferences by focusing only on two elements once at a time. It allows to reduce uncertainty and hesitation while leading to the higher of consistency. The problem is that the definition of a preference relation does not imply any kind of consistency property, and the decision makers' preferences can be inconsistent [13]. Luckily, the lack of consistency can be quantified and monitored, and it has been used as a parameter to validate the final solution obtained after a consensus process $[11,24]$. In such a way, consensus approaches using both consistency and consensus measures to guide the consensus process have been presented in $[8,24,30]$. Here, a consensus/consistency level is usually calculated as a weighted aggregation of the consistency level and the consensus degree, and it is used as a control parameter to decide if the consensus process has to finish. 
It should be pointed out that the incorporation of other additional criteria in the consensus process, as, for instance, consistency measures, contributes to enrich the consensus processes and to achieve more adequate solutions in the GDM. For example, the use of the consistency measures avoids misleading solutions, which cannot be detected by the consensus approaches using only consensus degrees.

\section{Conclusions and Future Work}

In this contribution, we have reviewed the different consensus approaches based on soft consensus measures that have been proposed in the literature in which the consensus process is guided by a moderator. To do so, some basic concepts to understand the topic have been introduced, and both the pioneering and most relevant contributions on consensus approaches have been highlighted. In addition, several approaches of consensus in GDM according to different criteria have been analyzed.

In the future, it is worth continuing this research by studying the current trends in the development of consensus approaches and by bringing out several issues that could represent challenges to be faced.

Acknowledgements The authors would like to acknowledge FEDER financial support from the Project TIN2013-40658-P, and also the financial support from the Andalusian Excellence Project TIC-5991.

\section{References}

1. Alonso, S., Pérez, I.J., Cabrerizo, F.J., Herrera-Viedma, E.: A linguistic consensus model for web 2.0 communities. Applied Soft Computing 13(1), 149-157 (2013)

2. Ben-Arieh, D., Chen, Z.: Linguistic-labels aggregation and consensus measure for autocratic decision making using group recommendations. IEEE Transactions on Systems Man and Cybernetics - Part A: Systems and Humans 36(3), 558-568 (2006)

3. Bezdek, J., Spillman, B., Spillman, R.: A fuzzy relation space for group decision theory. Fuzzy Sets and Systems 1(4), 255-268 (1978)

4. Bryson, N.: Group decision-making and the analytic hierarchy process: Exploring the consensus-relevant information content. Computers \& Operations Research 23(1), 27-35 (1996)

5. Butler, C.T., Rothstein, A.: On conflict and consensus: A handbook on formal consensus decision making. Tahoma Park (2006)

6. Cabrerizo, F.J., Moreno, J.M., Pérez, I.J., Herrera-Viedma, E.: Analyzing consensus approaches in fuzzy group decision making: Advantages and drawbacks. Soft Computing 14(5), 451-463 (2010)

7. Cabrerizo, F.J., Heradio, R., Pérez, I.J., Herrera-Viedma, E.: A selection process based on additive consistency to deal with incomplete fuzzy linguistic information. Journal of Universal Computer Science 16(1), 62-81 (2010)

8. Cabrerizo, F.J., Pérez, I.J., Herrera-Viedma, E.: Managing the consensus in group decision making in an unbalanced fuzzy linguistic context with incomplete information. KnowledgeBased Systems 23(2), 169-181 (2010) 
9. Chen, S.J., Hwang, C.L.: Fuzzy multiple attributive decision making: Theory and its applications. Springer, Berlin (1992)

10. Chen, X., Zhang, H., Dong, Y.: The fusion process with heterogeneous preference structures in group decision making: A survey. Information Fusion 24, 72-83 (2015)

11. Chiclana, F., Herrera-Viedma, E., Herrera, F., Alonso, S.: Some induced ordered weighted averaging operators and their use for solving group decision making problems based on fuzzy preference relations. European Journal of Operational Research 182(1), 383-399 (2007)

12. Coch, L., French, J.R.P.: Overcoming resistance to change. Human Relations 1(4), 512-532 (1948)

13. Cutello, V., Montero, J.: Fuzzy rationality measures. Fuzzy Sets and Systems 62(1), 39-54 (1994)

14. Dubois, D., Prade, H., Testemale, C.: Weighted fuzzy pattern matching. Fuzzy Sets and Systems 28(3), 313-331 (1988)

15. Dubois, D., Koning, J.L.: Social choice axioms for fuzzy set aggregation. Fuzzy Sets and Systems 43(3), 257-274 (1991)

16. Fedrizzi, M., Kacprzyk, J., Zadrozny, S.: An interactive multi-user decision support system for consensus reaching processes using fuzzy logic with linguistic quantifiers. Decision Support Systems 4(3), 313-327 (1988)

17. Fedrizzi, M., Pasi, G.: Fuzzy logic approaches to consensus modeling in group decision making. In: Ruan, D., Hardeman, F., Van Der Meer, K. (Eds) Intelligent Decision and Policy Making Support Systems, pp. 19-37. Springer-Verlag, Berlin-Heidelberg (2008)

18. French, J.R.P.: A formal theory of social power. Psychological Review 63(3), 181-194 (1956)

19. Fodor, J., Roubens, M.: Fuzzy preference modeling and multicriteria decision support. Kluwer, Dordrecht (1994)

20. Grabisch, M., Marichal, J.-L., Mesiar, R., Endre, P.: Aggregation functions (Encyclopedia of Mathematics and its Applications). Cambridge University Press, New York (2009)

21. Grabisch, M., Labreuche, C.: Fuzzy measures and integrals in MCDA. In: Greco, S., Ehrgott, M., Figueira, J.R. (Eds) Multiple Criteria Decision Analysis, pp. 553-603. Springer, New York (2016)

22. Harary, F.: On the measurement of structural balance. Behavioral Science 4(4), 316-323 (1959)

23. Herrera, F., Herrera-Viedma, E., Verdegay, J.L.: A model of consensus in group decision making under linguistic assessments. Fuzzy Sets and Systems 78(1), 73-87 (1996)

24. Herrera, F., Herrera-Viedma, E., Verdegay, J.L.: A rational consensus model in group decision making using linguistic assessments. Fuzzy Sets and Systems 88(1), 31-49 (1997)

25. Herrera, F., Herrera-Viedma, E., Verdegay, J.L.: Linguistic measures based on fuzzy coincidence for reaching consensus in group decision making. International Journal of Approximate Reasoning 16(3-4), 309-334 (1997)

26. Herrera, F., Herrera-Viedma, E.: Linguistic decision analysis: Steps for solving decisions problems under linguistic information. Fuzzy Sets and Systems 115(1), 67-82 (2000)

27. Herrera, F., Alonso, S., Chiclana, F., Herrera-Viedma, E.: Computing with words in decision making: Foundations, trends and prospects. Fuzzy Optimization and Decision Making 8(4), 337-364 (2009)

28. Herrera-Viedma, E., Herrera, F., Chiclana, F.: A consensus model for multiperson decision making with different preference structures. IEEE Transactions on Systems, Man, and Cybernetics - Part A: Systems and Humans 32(3), 394-402 (2002)

29. Herrera-Viedma, E., Martinez, L., Mata, F., Chiclana, F.: A consensus support system model for group decision-making problems with multigranular linguistic preference relations. IEEE Transactions on Fuzzy Systems 13(5), 644-658 (2005)

30. Herrera-Viedma, E., Herrera, F., Alonso, S.: Group decision-making model with incomplete fuzzy preference relations based on additive consistency. IEEE Transactions on Systems, Man and Cybernetics - Part B: Cybernetics 37(1), 176-189 (2007)

31. Herrera-Viedma, E., Cabrerizo, F.J., Kacprzyk, J., Pedrycz, W.: A review of soft consensus models in a fuzzy environment. Information Fusion 17, 4-13 (2014) 
32. Kacprzyk, J.: Group decision-making with a fuzzy majority via linguistic quantifiers. Part I: A consensory-like pooling. Cybernetics and Systems: An International Journal 16(2-3), 119-129 (1985)

33. Kacprzyk, J.: Group decision-making with a fuzzy majority via linguistic quantifiers. Part I: A competitive-like pooling. Cybernetics and Systems: An International Journal 16(2-3), 131-144 (1985)

34. Kacprzyk, J.: Group decision making with a fuzzy linguistic majority. Fuzzy Sets and Systems 18(2), 105-118 (1986)

35. Kacprzyk, J.: On some fuzzy cores and 'soft' consensus measures in group decision making. In: Bezdek, J.C. (Ed) The Analysis of Fuzzy Information, pp. 119-130. CRC Press, Boca Raton (1987)

36. Kacprzyk, J., Fedrizzi, M.: A 'soft' measure of consensus in the setting of partial (fuzzy) preferences. European Journal of Operational Research 34(3), 316-325 (1988)

37. Kacprzyk, J., Fedrizzi, M.: A 'human-consistent' degree of consensus based on fuzzy logic with linguistic quantifiers. Mathematical Social Sciences 18(3), 275-290 (1989)

38. Kacprzyk, J., Fedrizzi, M., Nurmi, H.: Group decision making and consensus under fuzzy preferences and fuzzy majority. Fuzzy Sets and Systems 49(1), 21-31 (1992)

39. Kacprzyk, J., Zadrozny, S., Ras, Z.W.: How to support consensus reaching using action rules: a novel approach. International Journal of Uncertainty, Fuzziness and Knowledge-Based Systems 18(4), 451-470 (2010)

40. Loewer, B.: Special issue on consensus. Synthese 62(1), 1-122 (1985)

41. Loewer, B., Laddaga, R.: Destroying the consensus. Synthese 62(1), 79-96 (1985)

42. Mata, F., Martinez, L., Herrera-Viedma, E.: An adaptive consensus support model for group decision-making problems in a multigranular fuzzy linguistic context. IEEE Transactions on Fuzzy Systems 17(2), 279-290 (2009)

43. Narukawa, Y., Torra, V.: Fuzzy measures and integrals in evaluation of strategies. Information Sciences 177(21), 4686-4695 (2007)

44. Orlovski, S.A.: Decision-making with a fuzzy preference relation. Fuzzy Sets and Systems 1(3), 155-167 (1978)

45. Pawlak, A.: Information systems theoretical foundations. Information Systems 6(3), 205-218 (1981)

46. Pérez, I.J., Cabrerizo, F.J., Alonso, S., Herrera-Viedma, E.: A new consensus model for group decision making problems with non homogeneous experts. IEEE Transactions on Systems, Man, and Cybernetics: Systems 44(4), 494-498 (2014)

47. Pérez, L.G., Mata, F., Chiclana, F., Kou, G., Herrera-Viedma, E.: Modeling influence in group decision making. Soft Computing 20(4), 1653-1665 (2016)

48. Roubens, M.: Fuzzy sets and decision analysis. Fuzzy Sets and Systems 90(2), 199-206 (1997)

49. Saaty, T.L.: The analytic hierarchy process: Planning, priority setting, resource allocation. McGraw-Hill, New York (1980)

50. Saint, S., Lawson, J.R.: Rules for reaching consensus: A modern approach to decision making. Jossey-Bass (1994)

51. Spillman, B., Bezdek, J., Spillman, R.: Coalition analysis with fuzzy sets. Kybernetes 8(3), 203-211 (1979)

52. Tanino, T.: Fuzzy preference orderings in group decision making. Fuzzy Sets and Systems 12(2), 117-131 (1984)

53. Torra, V., Narukawa, Y.: Modeling decisions: Information fusion and aggregation operators. Springer-Verlag (2007)

54. Yager, R.R.: On ordered weighted averaging aggregation operators in multicriteria decision making. IEEE Transactions on Systems Man and Cybernetics 18(1), 183-190 (1988)

55. Zadeh, L.A.: Fuzzy sets. Information and Control 8(3), 338-353 (1965)

56. Zadeh, L.A.: A computational approach to fuzzy quantifiers in natural languages. Computers \& Mathematics with Applications 9(1), 149-184 (1983)

57. Zadrozny, S., Kacprzyk, J.: Issues in the practical use of the OWA operators in fuzzy querying. Journal of Intelligent Information Systems 33(3), 307-325 (2009) 